\title{
NUMERICAL MODELLING OF THE TFTR ICRH ANTENNAS
}

\author{
M. Kress*, Y. L. Ho, W. Grossmann, A. Drobot \\ Science Applications International Corporation \\ 1710 Goodridge Drive, McLean, VA 22102 \\ DE92 002061 \\ D. B. Batchelor, P. M. Ryan, M. Carter \\ Oak Ridge National Laboratory \\ CONF-910869--18

\begin{abstract}
A general purpose 3D electromagnetic field solver code, ARGUS, is being used to analyze the TFTR Antennas. To date, the vacuum radiation patterns produced by the bay $M$ and $L$ antennas have been obtained and reported. Recent work has concentrated on antenna performance comparison and understanding the role of geometry on performance (e.g, the impact of end-effects on current 2D models). Additional diagnostics such as evaluation of phase velocity and strap inductance are being implemented to enhance our understanding and to better compare with measurements.
\end{abstract}

\section{INTRODUCTION}

To date, the most useful theoretical analyses of ICRH antennae and rf plasma heating have been carried out using combinations of one- and two-dimensional plasma-antenna models. These studies (see e.g. Ref. 1) have provided valuable insights to antenna designers and have also been successful in analyzing the results of cur ent ICRH experiments. In order to design, analyze and evaluate antennae proposed for future fusion reactors-where conditions will be extreme-we will require more robustness and fidelity in the model predictions. To this end, SAIC is developing a fully 3-D simulation capability that allows detailed modelling of realistic ICRF antennas. Our research thus far has concentrated on vacuum operations. The goals are to validate the simulation modules in place (through comparison with experiments), to learn to better interpret our results, and to investigate features of the vacuum field solutions which may directly impact antennae performance with plasma. This paper presents the present status of simulation efforts associated with the ICRF antennas on the PPPL TFTR experiment: namely, the Bay $L$ and $M$ antennas (early results have been presented elsewhere ${ }^{2-4}$ ).

*Permanent address: City University of New York, Staten Island, NY

\section{MASTER}

DISTRIBUTION OF THIS DOCUMENT IS UNLIMITED 


\section{THE ARGUS SIMULATION CODE}

The ARGUS code c-6 $^{5}$ is a general-purpose three-dimensional simulation code designed to handle problems involving complex geometric structures. Nonuniform grids are used in all three dimension to efficiently capture fine structural details. Presently, the code computes electrostatic and electromagnetic fields, the eigenmodes of rf structures, and can perform PIC simulation in either a time-dependent mode or a steady-state mode. The PIC modules include multiple particle species, the Lorentz equations of motion, phenomenological terms for elastic and inelastic scattering, and algorithms for the creation of particles by emission from material surfaces, injection onto the grid, and ionization. Imposition of the boundary condition is highly flexible: either metallic walls, periodic boundaries, or port conditions describing in-going or outgoing radiation can be imposed. Internally, material structures can be defined on the grid as regions of specified material properties (e.g., perfectly conducting, dielectric constant, resistivity, or permeability).

ARGUS has been successfully applied to and benchmarked with a broad spectrum of problems. The electrostatic solvers have been used as stand-alone codes to analyze the dc component of the fields in a crossfield amplifier tube. They have also been used with the PIC module to carry out steady-state simulations of electron guns, depressed electron collectors, ion guns, and low-energy electrostatic ion accelerators. The electromagnetic modules in ARGUS have been used to carry out coldtest calculations for microwave coupled-cavity tubes and accelerator structures. Calculations with lossy materials have been carried out to model severs in a microwave amplifier and high-order-mode dampers in a gyroklystron amplifier circuit.

\section{CODE VALIDATION STUDIES}

Initial attempts at validating ARGUS for application to ICRF antennas were concentrated on the University of Wisconsin model antenna2-3. Figure 1 (a) shows a numerical grid representation of half the antenna structure as configured on the test-stand. In Figure 1(b), we compare the computed versus measured vacuum toroidal if $B$ field taken above the strap surface as a function of toroidal position. The agreement is excellent. Notably, this work also revealed that current flowing in the support structures (such as the backplane) can significantly affect the antenna radiation pattern, thus reaffirming the value of full $3 \mathrm{D}$ simulations. 

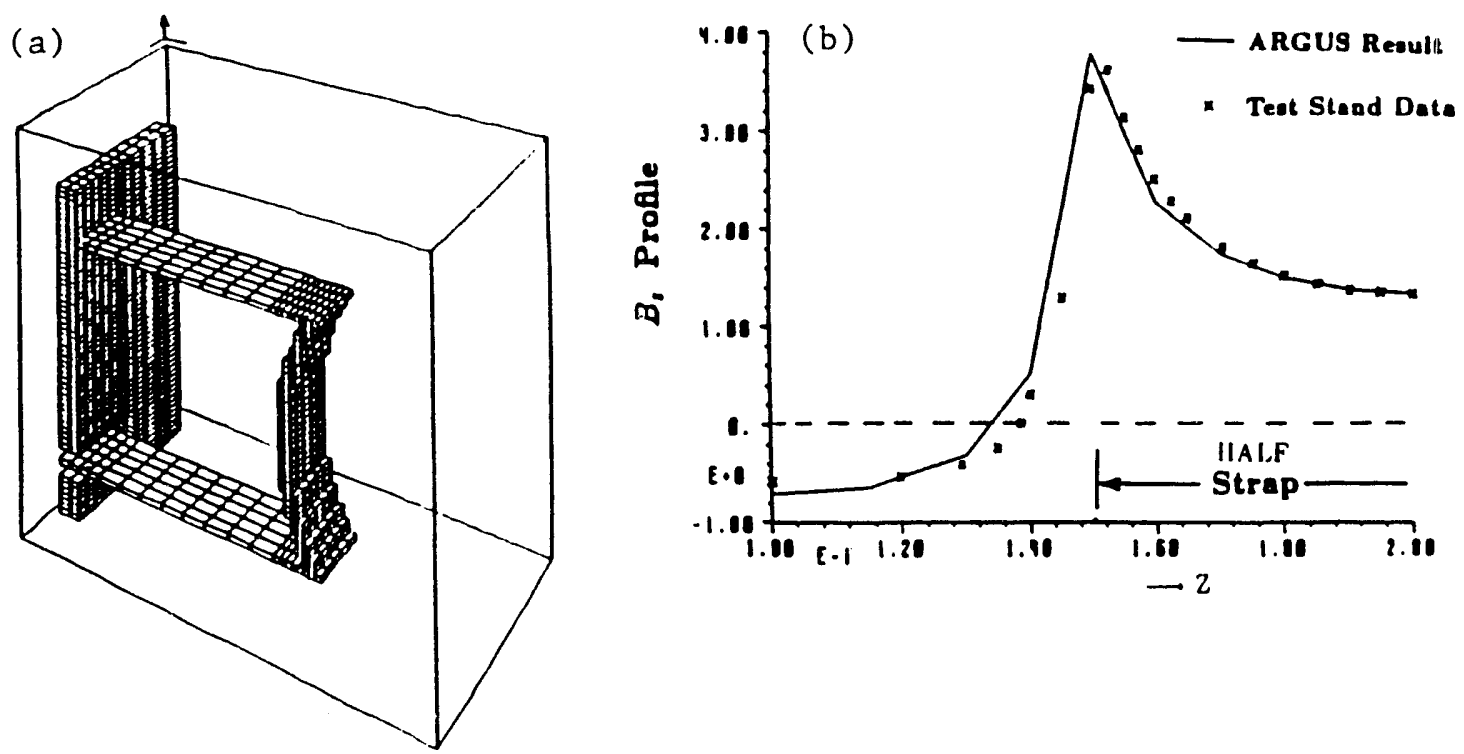

Fig. 1. Comparison between ARGUS and experiment: (a) numerical grid representation of half the University of Wisconsin antenna;

(b) computed versus measured vacuum toroidal magnetic field, $B_{Z}$, taken above the strap surface as a function of toroidal, $z$, position.

\section{TFTR ANTENNA SIMULATION}

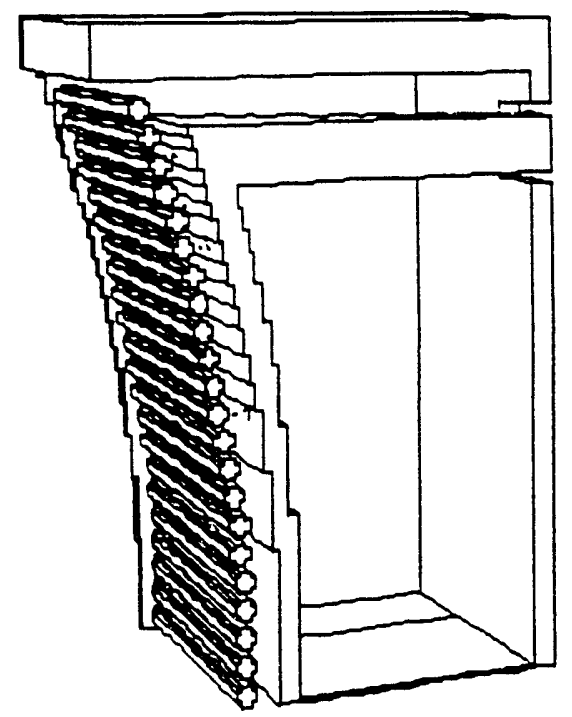

Fig. 2. Numerical grid representation of the TFTR bay-L antenna.
Recently, we have used ARGUS to model the ICRH antennas at TFTR. The numerical grid representation of the double strap Bay $M$ antenna is shown in Figure 2. Only the upper right quadrant is modeled; symmetry conditions are used to reduce computing requirements. The figure shows a cutaway of the antenna with current straps, Faraday rods, end plates and center septum. Not shown, but included in the model, are the side walls. Power leads to the antenna are simulated through a gap in the back plate fed with an applied voltage at the actual frequency (typically driven at $47 \mathrm{Mhz}$ ). ARGUS calculates all rf electric and magnetic field components, current distribution in straps, Faraday rods and walls. Derived quantities such as strap current phase velocity and mutual inductances (multiple straps) can be calculated from the ARGUS output. 
We now present results from an analysis of the near field power radiated away in vacuum from the antenna. In particular, we evaluate the Poynting flux through a vertical flat plane separated by two different distances from the front of the current strap $(-10.5 \mathrm{~cm}$ and $12 \mathrm{~cm}$ from the center short). Figure 3 compares their temporal evolution for the 0 -phasing (two straps driven in phase) case. Note that the Poynting flux rapidly decrease with distance away from the antenna face. This indicates that spatial Fourier analysis of the power spectra on a surface will sensitively depend on the shape of the surface; great care is required when interpreting those results $2-4$.

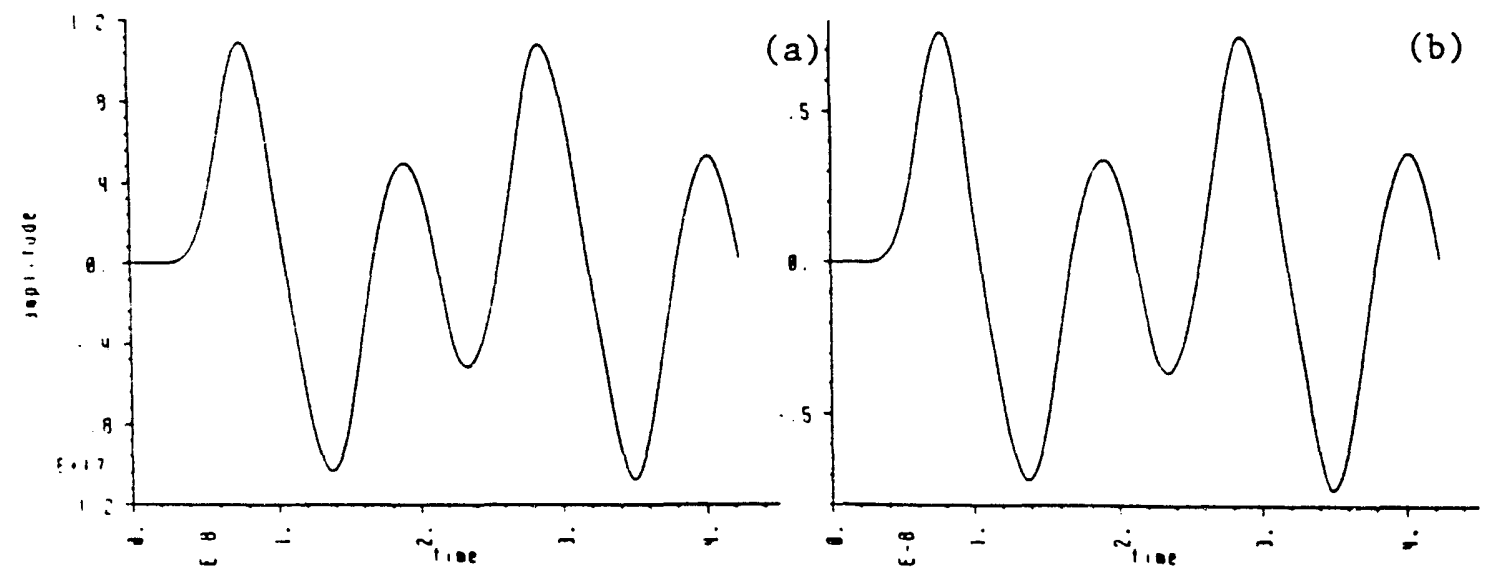

Fig. 3. Comparison of Poynting flux through a vertical flat plane at (a) approximately $10.5 \mathrm{~cm}$ and (b) approximately $12 \mathrm{~cm}$ in front of the model bay-L current strap. The distances are measured from the center short.

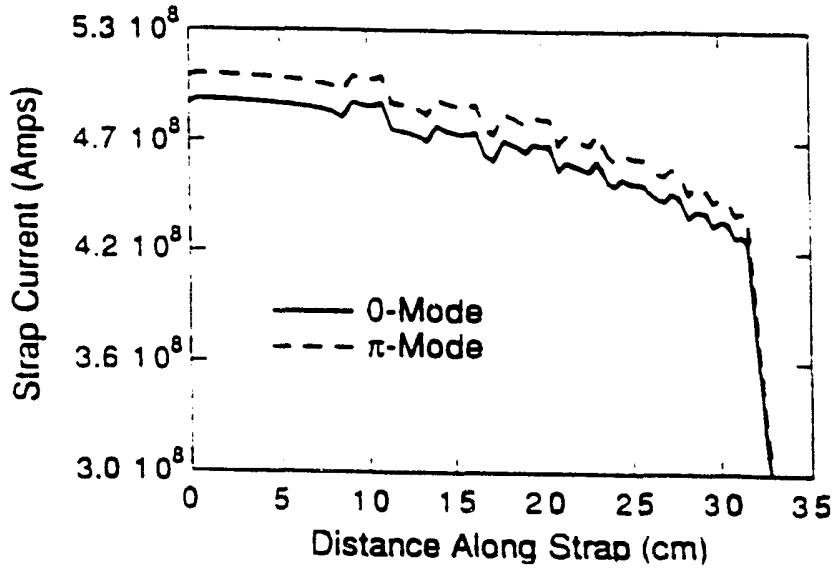

Fig. 4. Current distributions along the front strap of the bay-L antenna. The amplitude in amps do not represent realistic current levels.
From the ARGUS computed field solutions, we can indirectly evaluate the current phase velocity and strap inductances. The phase velocity is obtained by fitting a cosine function to the current distribution along the strap. Figure 4 shows the strap current distribution for both zero-and $\pi$-mode operations along the curved front section of the strap starting from the center short. The rapid fall-off of the current at the right side of the curves is due to the 
change in direction of the strap current; there, the vertical front section is connected to the horizontal feed. The small scale fluctuations are due to grid discretization. From this current distribution, the phase velocity is estimated to be $0.57 c$, where $c$ is the speed of light in vacuum.

For the strap inductance evaluations, we use the relationship between strap current and total magnetic energy, $W$, where

$$
W=0.5 L\left[I_{1}^{2}+I_{2}^{2}\right]+M I_{1} I_{2}
$$

Here, $I_{1}$ and $I_{2}$ are the currents flowing the two straps respectively, $L$ is the self-inductance and $M$ is the mutual-inductance. Using both zeromode and $\pi$-mode results, we obtain two linearly dependent equations from which we solve for the inductances. For the model Bay $L$ antenna, the results are $L \approx 2.4 \times 10-7$ henry, and $M \approx 4.9 \times 10-9$ henry. There is, however, one caveat: the current is nonuniform along the strap. For these calculations, we opted to use the current value at the backplane. Future calculations of the inductances will require running the simulation in a steady state such that the current distribution will be uniform.

\section{CONCLUSION}

The utility of ARGUS for modeling future reactor configurations lies in the ability to use a common geometry data base for if performance, and for structural and thermal analyses. While we have demonstrated some of its existing capability here, significant diagnostic/code upgrades and further validation studies are underway. Briefly, ARGUS results will be benchmarked against measurements done on mockup DIII-D and TFTR antennas at ORNL. An idealized antenna model is being used to elucidate the role of end-effects on current 2D models. A frequency domain solver is being implemented that will significantly reduce the computational time, and facilitate the addition of the plasma simulation module. And for diagnostics, we are installing the capability to evaluate the $k \mid 1$ power spectrum on a flux surface.

\section{ACKNOWLEDGMENT}

This work was supported by the U.S Department of Energy

\section{KEFERENCES}

1. D.B.Batchelor, Presented at the "Workshop on ICRF Physics and Design", Boulder, CO, (1991). 
2. W. Grossmann, et.al, in Proc. of $16^{\text {th }}$ Eur. Conf. on Contr. Fusion and Plasma Physics, Venice, Italy (1989).

3. D. A. D'Ippolito, et.al., in Proc. of $13^{\text {th }}$ Intl. Conf. on Plasma Physics and Controlled Nuclear Fusion Research, Paper IAEA-CN-53/E-31, Washington, DC, (1990).

4. W. Grossmann et. al. Bull. Am. Phys. Soc. 35. 1965 (1990).

5. A. Mankofsky, in Linear Accelerator and Beam Optics Codes, C. R. Eminheizer, ed., A.I.P. Conf. Proc. No. 177 (AIP, New York, 1988), p. 137ff.

6. C. L. Chang et. al. in Proc. of the Conf. on Computer Codes and the Linear Accelerator Community, R. K. Cooper, ed. (Los Alamos National Laboratory, January 22-25, 1990, LA-11857-C), p. 27. 

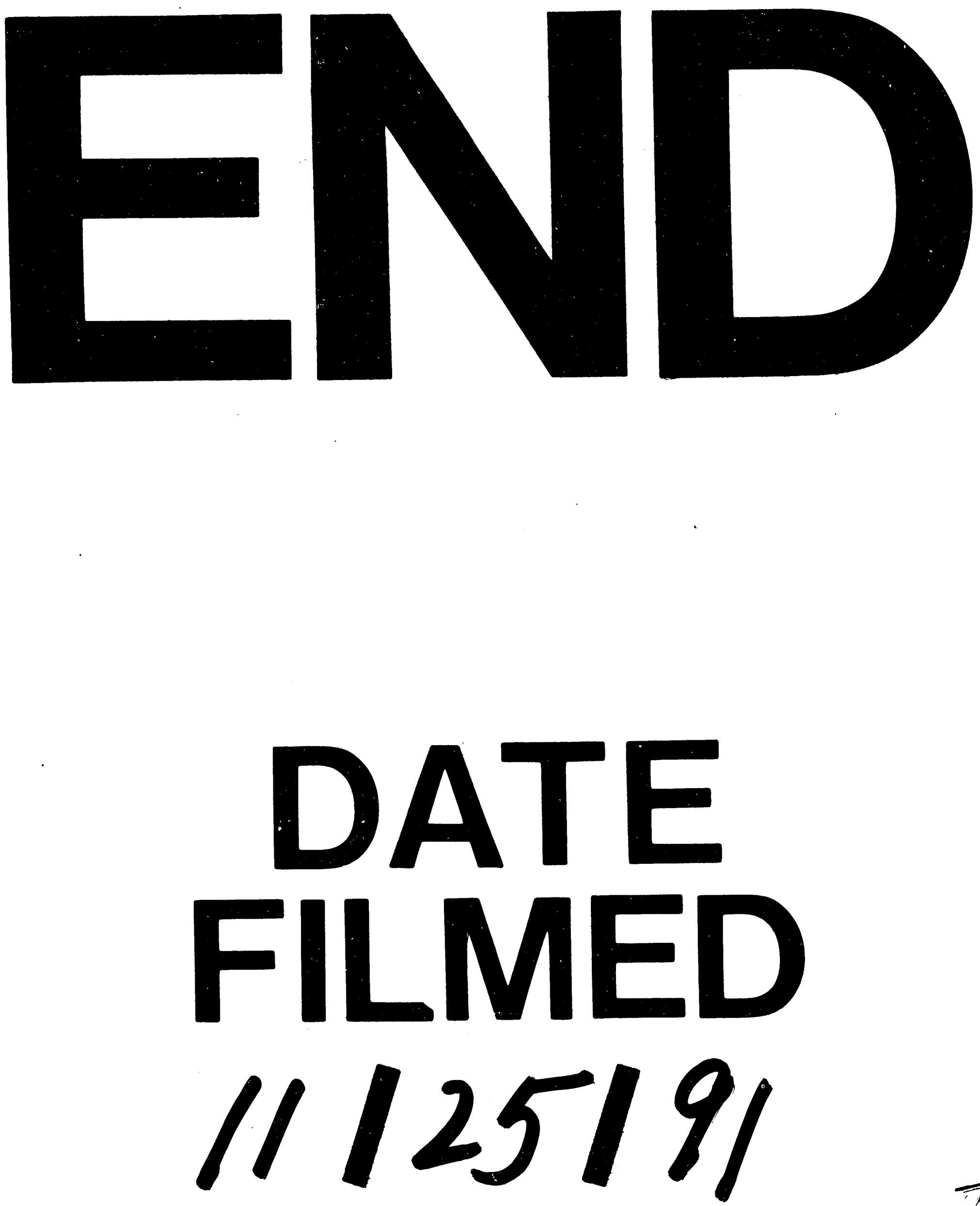
